\title{
Ureteroscopy and holmium laser lithotripsy: Is this procedure safe in pregnant women with ureteral stones at different locations?
}

\author{
Senol Adanur ${ }^{1}$, Tevfik Ziypak ${ }^{1}$, Fevzi Bedir ${ }^{1}$, Turgut Yapanoglu ${ }^{1}$, Hasan Riza Aydın ${ }^{2}$, \\ Mehmet Yilmaz ${ }^{3}$, Mehmet Aksoy ${ }^{4}$, İsa Ozbey ${ }^{1}$ \\ ${ }^{1}$ Department of Urology, Medical Faculty, Ataturk University; \\ 2 Department of Urology, Akçaabat Haçkalı Baba Hospital, Trabzon; \\ ${ }^{3}$ Department of Obstetrics and Gynecology, Medical Faculty, Ataturk University; \\ ${ }^{4}$ Department of Anaesthesiology and Reanimation, Medical Faculty, Ataturk University.
}

\begin{abstract}
Summary Objectives: The aim of this study was to assess the safety and effectiveness of ureteroscopy and Holmium: Yttrium-Aluminum-Garnet lithotripsy for the treatment of ureteral stones with different localizations in symptomatic pregnant women. Methods: A retrospective analysis was performed on 19 pregnant patients referred to our center between January 2005 and December 2012 with symptomatic hydronephrosis requiring surgical intervention. $7.5 \mathrm{~F}$ and $9.5 \mathrm{~F} \mathrm{semi-}$ rigid ureterorenoscopy with Holmium laser lithotripsy was used for treatment in all patients. Complications were stratified according to modified Clavien criteria.

Results: The mean age of patients was 25.4 (18-41) years, and the mean gestation duration was 24.8 (7-33) weeks. Six cases $(31.5 \%)$ had a history of stone. Solitary kidney secondary to previous nephrectomy was observed in 2 patients and 1 patient had a hypoplastic kidney.

Abdominal ultrasonography was used as the main diagnostic tool. Mean stone size was $9.2 \mathrm{~mm}$ (6-13). The location of the stones was the lower, middle, and upper ureter in 8 (42.1\%), 5 (26.3\%) and 6 (31.5\%) cases, respectively.

All stones were fragmented with Holmium laser lithotripsy. Of the 19 patients, 11 (57.8\%) required doublr J stent insertion peroperatively. Intraoperative urological and obstetric complications were not observed. Postoperatively two complications were noted. According to Clavien criteria a complication was level 1 , and the other was level 2. Conclusions: For treatment of pregnant women with symptomatic ureteral stones in every location, Holmium laser lithotripsy with a semirigid ureteroscopy can be used as judicious treatment. This approach is effective and safe with an acceptable complication rate.
\end{abstract}

KEY WORDS: Urinary calculi; Pregnancy; Ureteroscopy; Laser lithotripsy.

Submitted 12 November 2014; Accepted 5 October 2013

\section{INTRODUCTION}

Incidence of symptomatic urinary stones has been reported as 1/200-1/2500 (1). Renal colic is the most frequent non-obstructive cause of hospitalization during pregnancy with $80-90 \%$ of urinary stones in pregnant women diagnosed after the first trimester $(2,3)$.

Fifty to eighty per cent of pregnant patients with symptomatic stones tend to pass their stones spontaneously when treated conservatively with analgesics and hydration $(1,4)$. Surgical intervention might be required in the presence of persistent obstruction or intractable pain refractory to analgesics which is the case in nearly one third of the patients (5). Advances in fibre optic technology lead to the design of new ureteroscopes, which allow atraumatic access into upper urinary tract (6) and Holmium: YtriumAluminium-Garnet (YAG) laser lithotripsy demonstrated to be able to fragment all types of stone with a certain degree of safety (7). These technical advances in laser lithotiptors and endoscopes offer the urologists an opportunity to safely treat urotlithiasis in pregnant women. In this study, we aimed to retrospectively analyze the effectiveness of the holmium: YAG laser lithotriptor with semi-rigid ureteroscope in the management of ureteral stones at different locations in pregnant women.

\section{Materials AND Methods}

In this study we retrospectively evaluated 19 pregnant women who presented to our clinics with the initial diagnosis of symptomatic hydronephrosis between January 2005 and December 2012. These patients whose renal colic and progressive hydronephrosis did not resolve despite conservative treatment during follow-up period underwent ureterorenoscopy (URS) for the detection and treatment of their stones. The medical files of these patients were retrospectively evaluated, and their ages, gestation trimesters, admission symptoms, diagnostic methods, degree of hydronephrosis, past history of urolithiasis or urological interventions, sizes and locations of stones and grades of complications based on modified Clavien criteria (8) were recorded. Diagnosis of ureteral obstruction was made based on actual clinical manifestations of the patients, their abdominal ultrasound (US)

No conflict of interest declared.

Archivio Italiano di Urologia e Andrologia 2014; 86, 2 
findings, and detection of haematuria in urinalysis. Obstructive stones were diagnosed in six (31.5\%) patients by US. All patients were also evaluated with whole blood count, urea and creatinine measurements, urine and (if required) blood cultures. The patients underwent obstetric consultations in order to rule out obstetric complications and determine their gestational weeks.

Conservative treatment such as intravenous fluid replacement and analgesics was started on all hospitalised patients. A third generation cephalosporin (ceftriaxone) was given in cases with infection. URS procedures were postponed in patients with active urinary system infection with documented bacterial growth in their urine cultures until their urine cultures became sterile.

Preoperative anaesthesiologic and obstetric consultations were required before URS procedure. All patients underwent preoperative intravenous antibiotic prophylaxis. URS procedures were performed under general anaesthesia without using halothane and nitric oxide. URS procedures were realised using a $7.5 \mathrm{~F}$ (Olympus Inc., Japan) or a 9.5 F (Karl Storz Inc., Germany) semi-rigid ureteroscope under direct endoscopic vision without fluoroscopic guidance. Ureters were accessed using 0.038 inch guide wires and in 3 patients balloon dilation of the stenotic distal end of the ureter was needed before intraureteral access. The stones were fragmented using holmium-YAG laser (Auriga, Wavelight Laser Tecnologie $A G$, Germany). A forcep was used for extraction of stone fragments. Cases with oedema secondary to an impacted stone, with presence of a concomitant renal stone or with ureteral trauma caused by URS necessitated double J stent application. During the early postoperative period, meticulous obstetric care was offered to all patients to ensure maternal and foetal well-being.

\section{RESULTS}

The mean age of the patients and the mean gestational age were 25.4 (18-41) years and 24.8 (7-33) weeks, respectively. Patient characteristics are listed in Table 1. Medical history of the patients revealed the existence of solitary kidney secondary to nephrectomy in 2, hypoplastich kidney in 1, and a past history of stone disease in $6(31.5 \%)$. The symptoms were renal colic in $14(73.6 \%)$ patients, fever in 2 (10.5\%) patients and microscopic ematuria/ pyuria was observed in 9 (47.3\%) patients. Eight (42.1\%) patients had right and eleven (57.9\%) had left side hydronephrosis. Four $(21 \%)$ patients had positive urine cultures. The mean stone size determined by pre-operative ultrasound in 6 patients was $9.2 \mathrm{~mm}(6-13 \mathrm{~mm})$. US detected grade I hydronephrosis in $2(10.5 \%)$, grade II in $10(52.9 \%)$ and grade III in 7 (36.8\%) patients. Ureteric stones were found in the distal ureter in 8 (42.1\%), middle in $5(26.3 \%)$ and proximal in $6(31.5 \%)$ patients. The stones of all patients were fragmented using holmium: YAG laser lithotriptor. Stone fragments of 9 patients were extracted using forceps, while those of other 10 patients were left to spontaneous passage. Intraureteral double- J stents were implanted in 11 (57.8\%) patients because of procedural oedema by impacted stone, concomitant renal stone, and intraureteral trauma during URS. Double- J stents of 7 patients were extracted 2-4 weeks after the first
Table 1.

Patient characteristic.

\begin{tabular}{|l|c|}
\hline Characteristic & Value $\mathbf{n}(\%)$ \\
\hline Patient (n) & 19 \\
\hline Age (years) & $25.4(18-41)$ \\
\hline Gestation (weeks) & $24.8(7-33)$ \\
\hline Laterality & $8(42.1)$ \\
$\quad$ Right & $11(57.9)$ \\
$\quad$ Left & $6(31.6)$ \\
\hline History of urolithiasis & $2(10.5)$ \\
\hline Soliter kidney & $1(5.2)$ \\
\hline Hypoplastic kidney & $14(73.6)$ \\
\hline Symptoms & $2(10.5)$ \\
Renal colic & $9(47.3)$ \\
$\quad$ Fever & $4(21)$ \\
$\quad$ Haematuria-microscopic pyuria & $6(31.6)$ \\
\hline Positive urine culture & $9.2(6-13)$ \\
\hline Stone diagnosed by ultrasonography & \\
\hline Stone size on ultrasonography (mm) & \\
\hline
\end{tabular}

procedure. Stents of 3 patients with concomitant renal stones smaller than $2 \mathrm{~cm}$ were extracted after successful shockwave lithitripsy (SWL) performed during the postnatal period. Double- J stent of one patient migrated on the 1st postoperative day. In 3 patients, following the procedure, an open end $5 \mathrm{~F}$ external ureteral catheter was inserted as a transitory stent. The stent was removed within 24 hours after the procedure. Details of the procedure and outcomes are presented in Table 2.

No intraoperative urological and obstetric complication was observed. During postoperative controls preterm uterine contractions $(\mathrm{n}=1)$, and urinary tract infection $(\mathrm{n}=1)$ developed. The patient who suffered from preterm uterine contractions was treated with tocolysis, and the other case with appropriate antibiotics. All newborns were healthy and delivered at term.

\section{Discussion}

Hydronephrosis induced by pregnancy is the most prevalent cause of urinary tract infection and can lead to

Table 2.

Details of procedure and outcome.

\begin{tabular}{|l|c|}
\hline & N (\%) \\
\hline Degree of hydronephrosis & $2(10.5)$ \\
Grade I & $10(52.9)$ \\
Grade II & $7(36.8)$ \\
Grade III & $6(31.6)$ \\
\hline Stone location & $5(26.3)$ \\
Proximal & $8(42.1)$ \\
Middle & $3(15.8)$ \\
Distal & $11(57.8)$ \\
\hline Ballon dilatation of ureteral orifice & $19(100)$ \\
\hline Double-J stent insertion & $1(5.2)$ \\
\hline Type of lithotriptor & $1(5.2)$ \\
Laser lithotripsy & \\
\hline Postoperative complication & Preterm uterin contraction \\
Urinary tract infection & \\
\hline
\end{tabular}


flank pain imitating renal colic. Upper urinary tract dilation is seen in $90 \%$ of pregnant women in their third trimesters, and it may persist up to 12 weeks postpartum. Dependent on the position of the foetus, it is more prevalent on the right side (9). During pregnancy, urolithiasis and episodes of renal colic can lead to the development of hydronephrosis and urinary stasis ensuing in pyelonephritis and urinary tract infection. These complications can eventually result in obstetric complications as spontaneous rupture, premature birth and the delivery of low birth weight new-borns (10).

Fifty to eighty per cent of pregnant women suffering from symptomatic urolithiasis can pass their stones thanks to conservative treatment modalities including intravenous hydration and analgesia $(1,4)$. In some patients more invasive treatment modalities may be required and in 20-30\% of pregnant women with urolithiasis surgical treatment may be required (11).

Conventional treatment of obstructed ureter can be accomplished by placement of a percutaneous nephrostomy (PCN) tube or insertion of an ureteral stent. However, placement of a PCN tube and of a ureteral stent in the early stages of the pregnancy can be associated with some risks as incrustation, patient's discomfort and urinary tract infection (12-14). Because of physiological hydronephrosis developed during pregnancy, ureteral stents can migrate more frequently (15).

Recent advances in surgical technology have revolutionised the designs of semi-rigid and flexible ureteroscopes leading to increased use of URS in pregnant women. Miniaturisation of ureteroscopes, and advances in intracorporeal lithotripsy technology ensure successful intraureteral access and management of stones at any level of the urinary tract without any traumatic complication (16). Since collecting system and ureter undergo physiological dilation in pregnancy, ureteroscopic procedures can be achieved without need for dilatation of the ureteral orifice (17-19). However, Rana et al. (12) reported that they had performed ureteral dilatations in 5 (26\%) of their 19 pregnant women whereas in another study, Bozkurt et.al (20) resorted to ureteral dilatation in $5(15.6 \%)$ of their 32 patients. We also accomplished URS procedures with the indication of symptomatic ureteral stone in $3(15.7 \%)$ of 19 patients only after ureteral dilatation.

In pregnancy, all types of intracorporeal lithotriptors have been used. Although obstetric complications related to different types of lithotriptors have been underestimated in published reports, there are concerns about unknown harmful foetus-related effects of URS procedures induced by electrothermal energy emitted by certain lithotriptors (21). Electrohydraulic lithotriptors (EHL) have the narrowest margin of safety because of their extremely high hydraulic pressure imposed on the ureteral wall (16). Relatively safe applications of ultrasonic lithotriptors have been demonstrated as consequence of their limited impact on adjacent structures (22). However, the impact of higher frequency vibrations on foetal organs has not been fully yet acknowledged and possible risks related to the hearing ability of the foetus have been advocated by some Authors (19). These concerns have led to the development of devices such as holmium laser, pulsed-dye laser or pneumatic lithotriptors that focus their energy on a localised area with minimal energy dispersion. Pneumatic lithotriptors can be used only with a semi-rigid ureteroscope because of their rigid probes. On the other hand, pulseddye laser lithotriptor cannot fragment stones with hard composition (23). As a result of continuous technological advances, a compact laser system was developed, which emits highly water-absorbable shock waves at $2140 \mathrm{~nm}$ wavelength. Holmium: YAG laser disperses its energy via a flexible fibre, which facilitates intracorporeal lithotripsy into collecting system. Holmium laser lithotriptor can be safely used if activated $0.5-1.0 \mathrm{~mm}$ away from the ureteral wall (24). Holmium laser lithotriptor is advantageous over other lithotriptors in that it can be used with both semi-rigid and flexible ureteroscopes, and has the capacity to fragment all types of stones with varying compositions (23). Other advantages of Holmium laser lithotriptors, can be enumerated including their ability to fragment the stones into relatively small particles when compared with other types of lithotriptors. These small stone particles can be easily eliminated from the collecting system without the need for stone basket or grasper for their extraction (25). When compared with EHL or pneumatic lithotriptors, Holmium laser lithotriptors create weak shock waves, which decrease the probability of retropulsion of stones and/or stone particles $(25,26)$. Laing et al. (27) reported a review series consisting of 116 pregnant women who underwent ureteroscopy for ureteral stone treatment: Holmium laser was used with success in 27 (23\%) patients and a pneumatic lithotriptor in 21 (18\%) patients. Bozkurt et al. (20) had detected ureteral stones in 27 of 32 patients after URS procedures performed for the management of ureteral stones and used Holmium laser lithotriptor in 17 patients for the fragmentation of stones. We successfully fragmented all stones localised in various segments of the ureter using Holmium laser lithotriptors in 19 patients who had undergone URS procedures because of progressive hydronephrosis, and uncontrolled renal colic. Fragmented stone particles in 9 patients were extracted using stone forceps and very small stone fragments in 10 patients were left to spontaneous passage. Ureteral stents placed following URS procedures are adequate to drain obstructed ureters despite increased urinary urate, and calcium concentrations in pregnancy leading to higher risk of incrustation (12). Bozkurt et al. (20) reported placement of double- J ureteral stent in 19 (59.4\%) of 32 pregnant women after URS procedures. Rana et al. (12) also reported placement of double- J ureteral stents in 12 of (63\%) 19 pregnant patients who were relieved of obstructive ureteral stones using pneumatic lithotriptors under the guidance of ureterorenoscopy. We also inserted double- J ureteral stents in 11 (57.8\%) of 19 patients following URS procedures. Stents were placed with the aid of 9.5 F ureteroscopes under direct vision. However, in patients in whom we used $7.5 \mathrm{~F}$ ureteroscopes, firstly a guide wire was inserted and advanced through a ureteroscope under direct vision into the kidney, and then the position of the guide wire was confirmed by US. Double- J stents of 7 patients were removed within 2-4 weeks after the first procedure with the aid of a cystoscope under local anaesthesia. On the 1st postoperative day after the URS procedure double- J stent migrated in one patient who was monitored for occurrence of renal colic and development 
of pelvicalyceal dilation by periodic US examinations. Since renal colic and pelvicalyceal dilation did not occur, double- J stent was not re-implanted. Double-J stents of 3 patients were removed after they became stone-free following SWL in the postnatal period due to the presence of concomitant renal stone. No complication related to the implantation of double-J stents occurred. Complications related to URS procedure in pregnant women are not frequent. URS complication rates in pregnant and non-pregnant women are not different. In their meta-analysis consisting of 108 pregnant women who had undergone ureteroscopic stone management, Michelle et al. classified a total of 9 complications according to modified Clavien criteria (8), and reported 2 level 1, 6 level 2, and 1 level 3 complications (28). In our series a total of 2 patients developed complications consisting in preterm uterine contractions ( $\mathrm{n}=1)$ which were managed with tocolysis and urinary tract infection (UTI) $(n=1)$ which was treated with appropriate antibiotics. According to Clavien classification, these complications were rated level 1 and 2 , respectively.

\section{ConcLusion}

In conservative treatment of pregnant patients with refractory symptomatic stones at any ureteral location which cannot pass spontaneously, semi-rigid-URS can be used safely for diagnostic and therapeutic purposes. Holmium: YAG laser lithotripter, which is the most effective intracorporeal lithotriptor for all kinds of stones at every location in the urinary tract with semi-rigid URS, can be used successfully and safely in pregnant women with acceptable maternal and foetal complication rates.

\section{References}

1. Gorton E, Whitfield HN. Renal calculi in pregnancy. Br J Urol. 1997; 80 (Suppl. 1):4-9.

2. Rodriguez PN, Klein AS. Management of urolithiasis during pregnancy. Surg Gynecol Obstet. 1988; 166:103-106.

3. Swanson SK, Heilman RL, Eversman WG. Urinary tract stones in pregnancy. Surg Clin North Am. 1995; 75:123-42.

4. Parulkar BG, Hopkins TB, Wollin MR, et al. Renal colic during pregnancy: a case for conservative treatment. J Urol. 1998; 159:365-368.

5. Wein AJ, Kavoussi LR, Novick AC, et al. Campbell-walsh Urology. Matlaga BR, Lingeman JE(ed) Urinary Calculi During Pregnancy, 10th edn. Elsevier, Philadelphia, 2012; pp 1379-1382.

6. Conlin MJ, Marberger M, Bagley DH. Ureteroscopy. Development and instrumentation. Urol Clin North Am. 1997; 24:25-42.

7. Sofer M, Watterson JD, Wollin TA, et al. Holmium:YAG laser lithotripsy for upper urinary tract calculi in 598 patients. J Urol. 2002; 167:31-4.

8. Dindo D, Demartines N, Clavien PA. Classification of surgical complications: a new proposal with evaluation in a cohort of 6336 patients and results of a survey. Ann Surg. 2004; 240:205-13.

9. Boridy IC, Maklad N, Sandler CM. Suspected urolithiasis in pregnant women: imaging algorithm and literature review. AJR Am J Roentgenol. 1996; 167:869-75.

10. Negru I, Pricop C, Costachescu G.. Renal colic in pregnancy. Rev Med Chir Soc Med Nat Iasi. 2010; 114:439-44.

11. Biyani CS, Joyce AD. Urolithiasis in pregnancy. II: management. BJU Int 2002; 89:819-23.

12. Rana AM, Aquil S, Khawaja AM. Semirigid ureteroscopy and pneumatic lithotripsy as definitive management of obstructive ureteral calculi during pregnancy. Urology. 2009; 73:964-7.

13. Kavoussi LR, Albala DM, Basler JW, et al. Percutaneous management of urolithiasis during pregnancy. J Urol. 1992; 148:1069-71.

14. Jarrard DJ, Gerber GS, Lyon ES. Management of acute ureteral obstruction in pregnancy utilizing ultrasound-guided placement of ureteral stents. Urology. 1993; 42:263-7.

15. Kavoussi LR, Jackman SV, Bishoff JT. Re: Renal colic during pregnancy: a case for conservative treatment. J Urol. 1998; 160:837-8.

16. Zheng W, Denstedt JD. Intracorporeal lithotripsy. Update on technology. Urol Clin North Am. 2000; 27:301-13.

17. Drago JR, Rohner TJ Jr, Chez RA. Management of urinary calculi in pregnancy. Urology. 1982; 20:578-81.

18. Scarpa RM, De Lisa A, Usai E. Diagnosis and treatment of ureteral calculi during pregnancy with rigid ureteroscopes. J Urol. 1996; 155:875-7.

19. Ulvik NM, Bakke A, Høisaeter PA. Ureteroscopy in pregnancy. J Urol. 1995; 154:1660-3.

20. Bozkurt Y, Penbegul N, Soylemez H, et al. The efficacy and safety of ureteroscopy for ureteral calculi in pregnancy: our experience in 32 patients. Urol Res. 2012; 40:531-5.

21. Kroovand RL. Stones in pregnancy and in children. J Urol. 1992; 148:1076-8.

22. Howards SS, Merrill E, Harris S, Cohn J. Ultrasonic lithotripsy: laboratory evaluation. Invest Urol. 1974; 11:273-7.

23. Watterson JD, Girvan AR, Beiko DT, et al. Ureteroscopy and holmium:YAG laser lithotripsy: an emerging definitive management strategy for symptomatic ureteral calculi in pregnancy. Urology. 2002; 60:383-7.

24. Santa-Cruz RW, Leveillee RJ, Krongrad A. Ex vivo comparison of four lithotripters commonly used in the ureter: what does it take to perforate?. J Endourol. 1998; 12:417-422.

25. Teichman JM, Vassar GJ, Bishoff JT, et al. Holmium:YAG lithotripsy yields smaller fragments than lithoclast, pulsed dye laser or electrohydraulic lithotripsy. J Urol. 1998; 159:17-23.

26. Sofer, Denstedt, 2000. Sofer M, Denstedt J. Flexible ureteroscopy and lithotripsy with the holmium:YAG laser. Can J Urol. 2000; 7:952956.

27. Laing KA, Lam TB, McClinton S, et al. Outcomes of ureteroscopy for stone disease in pregnancy: results from a systematic review of the literature. Urol Int 2012; 89:380-6.

28. Semins MJ, Trock BJ, Matlaga BR. The safety of ureteroscopy during pregnancy: a systematic review and meta-analysis. J Urol. 2009; 181:139-43

\section{Correspondence}

Senol Adanur, MD - s.adanur61@hotmail.com

Tevfik Ziypak, MD

Fevzi Bedir, MD

Turgut Yapanoglu, MD

İsa Ozbey, MD

Department of Urology - School of Medicine - Ataturk University

25240 Erzurum, Turkey

Hasan Riza Aydın, MD

Department of Urology-kçaabat Haçkalı Baba Hospital, Trabzon, Turkey

Mehmet Yllmaz, MD

Department of Obstetrics and Gynecology, Medical Faculty

Ataturk University

Mehmet Aksoy, MD

Department of Anaesthesiology and Reanimation,

Medical Faculty Ataturk University, 25240 Erzurum, Turkey 